\title{
Paricalcitol Inhibits Wnt/ $\beta$-Catenin Signaling Pathway and Ameliorates Dermal Fibrosis in Bleomycin Induced Scleroderma Model
}

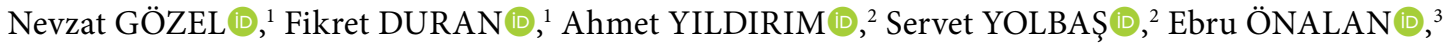 \\ İbrahim Hanifi ÖZERCAN(D, ${ }^{4}$ Süleyman Serdar KOCA(D ${ }^{2}$ \\ ${ }^{1}$ Department of Internal Medicine, Firat University Faculty of Medicine, Elazığ, Turkey \\ ${ }^{2}$ Department of Rheumatology, Firat University Faculty of Medicine, Elazı̆̆, Turkey \\ ${ }^{3}$ Department of Medical Biology, Firat University Faculty of Medicine, Elazığ, Turkey \\ ${ }^{4}$ Department of Pathology, Firat University Faculty of Medicine, Elazı̆̆, Turkey
}

\begin{abstract}
Objectives: This study aims to determine the prophylactic and therapeutic efficacy of inhibition of Wnt/ $\beta$-catenin signaling pathway with paricalcitol in an experimental scleroderma model created with bleomycin (BLM).

Materials and methods: Sixty female BALB/c mice (8-week old and weighing $25 \mathrm{~g}$ to $30 \mathrm{~g}$ ) were divided into six groups as prophylactic-early [control I (group 1)], sham I (group 2), paricalcitol I (group 3), therapeutic-late [control II (group 4)], sham II (group 5), and paricalcitol II (group 6) groups. Subcutaneous BLM (100 $\mu \mathrm{g} /$ day) injections were used to induce dermal fibrosis and paricalcitol $(0.3 \mu \mathrm{g} / \mathrm{kg} /$ day) was applied subcutaneously to BLM-injected mice during the first three weeks for preventive interventions and in the second three weeks for therapeutic interventions. Tissue samples were harvested for subsequent pathological and real-time polymerase chain reaction (RT-PCR) analysis. Tissue transforming growth factorbeta 1 (TGF- $\beta 1$ ), axin-1, and Wnt-2 messenger ribonucleic acid (mRNA) expressions were determined by RT-PCR.

Results: Repeated BLM applications increased the dermal inflammatory cell infiltration and dermal thickness, and led to dermal fibrosis, in both early and late stages. Similarly, TGF- $\beta 1$, axin-1, and Wnt-2 expressions were significantly increased in the sham groups compared to the own control group ( $p<0.05$ for all). Contrarily, prophylactic and therapeutic paricalcitol applications decreased the TGF- $\beta 1$, axin-1, and Wnt- 2 mRNA expressions compared to the own sham group ( $p<0.05$ for all). In addition, the regressions in dermal necro-inflammation and dermal fibrosis on pathological views were also observed in the paricalcitol applied groups.

Conclusion: In this model, increased axin-1 and Wnt-2 mRNA expressions suggest that Wnt/ $\beta$-catenin pathway is active in dermal fibrosis.

Keywords: Dermal fibrosis; paricalcitol; scleroderma; Wnt/ß-catenin pathway.
\end{abstract}

Scleroderma is a chronic inflammatory disease characterized by widespread fibrosis. ${ }^{1}$ The excessive production of extracellular matrix (ECM) molecules and their accumulations lead to fibrosis, although the pathogenesis of scleroderma is not fully described. ${ }^{2}$ Activated fibroblast, which is also known as myofibroblast, is the main source of ECM molecules.,4 Inflammatory cells lead to the activation of fibroblasts via producing several cytokines, adhesion molecules and growth factors, in addition to activated endothelial cells.
Transforming growth factor-beta (TGF- $\beta$ ) is the most famous actor at this process. ${ }^{5}$ After scleroderma fibroblasts are activated, they can also produce several cytokines, chemokines and growth factors, in addition to ECM molecules. Therefore, scleroderma fibroblasts perform autocrine and paracrine duties and it is accepted that they have different morphologic properties..$^{1-5}$

Wingless-type mouse mammary tumor virus integration site $(\mathrm{Wnt}) / \beta$-catenin signaling pathway

Received: September 26, 2017 Accepted: October 31, 2017 Published online: November 27, 2017

Correspondence: Nevzat Gözel, MD. Fırat Üniversitesi Tıp Fakültesi İç Hastalıkları Anabilim Dalı, 23119 Elazığ, Turkey. Tel: +90 424 - 2333555 e-mail: drngozel@hotmail.com

\section{Citation:}

Gözel N, Duran F, Yıldırım A, Yolbaş S, Önalan E, Özercan IH, et al. Paricalcitol Inhibits Wnt/B-Catenin Signaling Pathway and Ameliorates Dermal Fibrosis in Bleomycin Induced Scleroderma Model. Arch Rheumatol 2018;33(3):288-294. 
has roles on the embryogenesis, morphogenesis, cell-cell adhesion, and transcriptional cell differentiation. ${ }^{6}$ In non-canonical pathway, Wnt proteins do not bind to their transmembrane receptors and thus destruction complex is composed to degrade $\beta$-catenin. Conversely, Wnt proteins bind to receptors in canonical pathway, and thus, destruction complex is distributed. $\beta$-catenin is enhanced and activated in canonical pathway. Activated $\beta$-catenin is translocated into nucleus to induce the production of target genes which play a role on cell differentiation. ${ }^{7,8}$ Axin- 1 is a component of the destruction complex such as $\beta$-catenin, adenomatous polyposis coli, kinases glycogen synthase kinase- 3 alpha/beta and casein kinase -1 . Axin- 1 is a central scaffold protein for the destruction complex, thus it is the rate-limiting factor of the destruction complex. Degradation of axin- 1 leads to stabilization of $\beta$-catenin. ${ }^{7,8}$

The expressions of Wnt-2, Wnt-10 $\beta$ and Wnt-11 have been demonstrated to be increased in experimental scleroderma models which are tight-skin-1 mice and bleomycin (BLM) induced skin fibrosis. ${ }^{9,10}$ Moreover, it has been reported in patients with scleroderma that skin Wnt-1 and Wnt-11 expressions are increased and the expression of Dickkopf-related protein 1 (DKK1), an inhibitor of Wnt/ $\beta$-catenin signaling pathway, is decreased. ${ }^{11} \beta$-catenin production has been shown to increase in skin and lung samples of scleroderma patients. ${ }^{11,12}$ It has been reported that in vivo applications of Wnt-3 and Wnt- $10 \beta$ lead to dermal fibrosis. ${ }^{10-13}$ A subsequent in vitro study has demonstrated that Wnt-3 activates myofibroblast. ${ }^{14}$

Paricalcitol, 19-nor-1,25-hydroxivitamin D2 competitively inhibits the connection of T-cell factor (TCF-4), a transcription factor, to $\beta$-catenin as through vitamin D receptor (VDR), and thus, inhibits the activity of Wnt/ $\beta$-catenin signaling pathway. ${ }^{15-17}$ It has been documented that paricalcitol inhibits Wnt/ $\beta$-catenin signaling pathway via VDR, and hence, ameliorates nephropathy experimentally induced by adriamycin. ${ }^{17}$ Moreover, paricalcitol has been reported to block the transformation of epithelial cell to mesenchymal cell. ${ }^{18}$ Therefore, in this study, we aimed to determine the prophylactic and therapeutic efficacy of inhibition of Wnt/ $\beta$-catenin signaling pathway with paricalcitol in an experimental scleroderma model created with BLM.

\section{MATERIALS AND METHODS}

Sixty female BALB/c mice (8-week old and weighing $25 \mathrm{~g}$ to $30 \mathrm{~g}$ ) were included in the study, which was conducted in Firat University, Faculty of Medicine, between January 2015 and June 2015. The mice were divided into six groups as prophylactic-early [control I (group 1)], sham I (group 2), paricalcitol I (group 3), therapeutic-late [control II (group 4)], sham II (group 5), and paricalcitol II (group 6) groups (Figure 1). The upper back skin was shaved for subcutaneous injections. The study was approved by the Animal Care and Ethics Committee of the Firat University, Elazig, Turkey.

In groups 1 and 4, phosphate-buffered saline (PBS) was injected subcutaneously $(100 \mu \mathrm{L} /$ day $)$ to the shaved back skin. The remaining four groups received subcutaneously $100 \mu \mathrm{g}$ BLM (Bleocin; bleomycin hydrochloride; Nippon Kayaku, Tokyo, Japan), which was dissolved in PBS $(100 \mu \mathrm{L})$ to induce dermal fibrosis. ${ }^{19}$ BLM was injected to shaved upper back skin for three weeks in groups 2 and 3 , and for six weeks in groups 5 and 6 . In addition to BLM, paricalcitol $(0.3 \mathrm{\mu g} / \mathrm{kg} / \mathrm{day})$ was injected subcutaneously to dorsal front of neck for the first three weeks in group 3 (prophylactic) and the second three weeks in group 6 (therapeutic). ${ }^{18}$

Mice in groups 1, 2, and 3 were sacrificed at the end of the third week, while mice in groups 4, 5, and 6 were sacrificed at the end of the sixth week. After final experimental applications, the animals were sacrificed by cervical dislocation under anesthesia with ketamine hydrochloride, and the back skins were collected for further examinations. The harvested skin samples were divided into two parts. One part for histopathological examinations

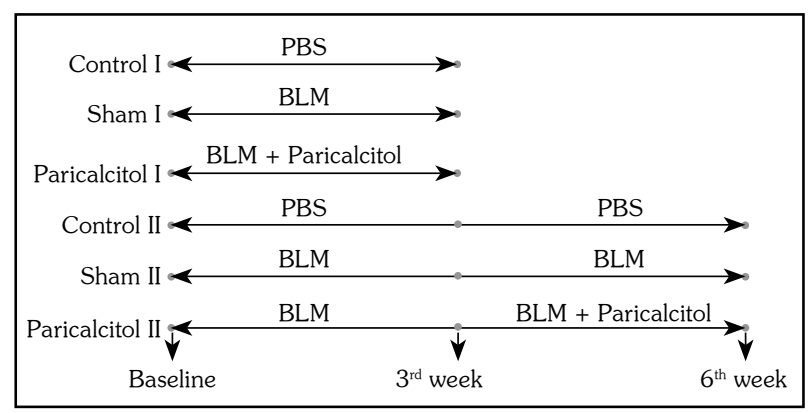

Figure 1. Schemes of experimental applications in study groups. PBS: Phosphate buffered saline; BLM: Bleomycin. 
was fixed with $10 \%$ formalin solution and embedded in paraffin, while the other part was stored immediately at $-80^{\circ} \mathrm{C}$ for real-time polymerase chain reaction $(\mathrm{PCR})$ analysis.

The paraffin embedded skin specimens were sectioned using a microtome. Subsequently, the skin specimens were stained with hematoxylin and eosin and Masson's trichrome. Dermal thickness was measured from the dermal-fat junction to epidermal-dermal junction and was determined from randomly selected five sites of two or more skin sections for each animal, by $40 \times, 100 \times$ and 200x magnifications. Histopathological examinations were performed under a light microscope (Olympus BX-50; Olympus America, Inc., Lake Success, NY, USA).
Total ribonucleic acid (RNA) was isolated from skin tissue using TRIzol reagent (Invitrogen, Carlsbad, CA, USA). Complementary deoxyribonucleic acid (cDNA) was generated by reverse-transcription of total RNA using cDNA synthesis kit (Invitrogen, Carlsbad, CA, USA). PCR reactions were prepared, in triplicate, and heated to $50^{\circ} \mathrm{C}$ for two minutes, followed by 40 cycles of denaturation at $95^{\circ} \mathrm{C}$ for 10 minutes, $95^{\circ} \mathrm{C}$ for 15 seconds, and $60^{\circ} \mathrm{C}$ for one minute. Standard curves were prepared for each target gene (Applied Biosystems, Foster City, CA, USA) as the endogenous reference in each sample. A real-time PCR analysis was performed with the ABI PRISM 7500 Fast Real Time PCR Instrument (Applied Biosystems, Foster City, CA, USA) using TaqMan
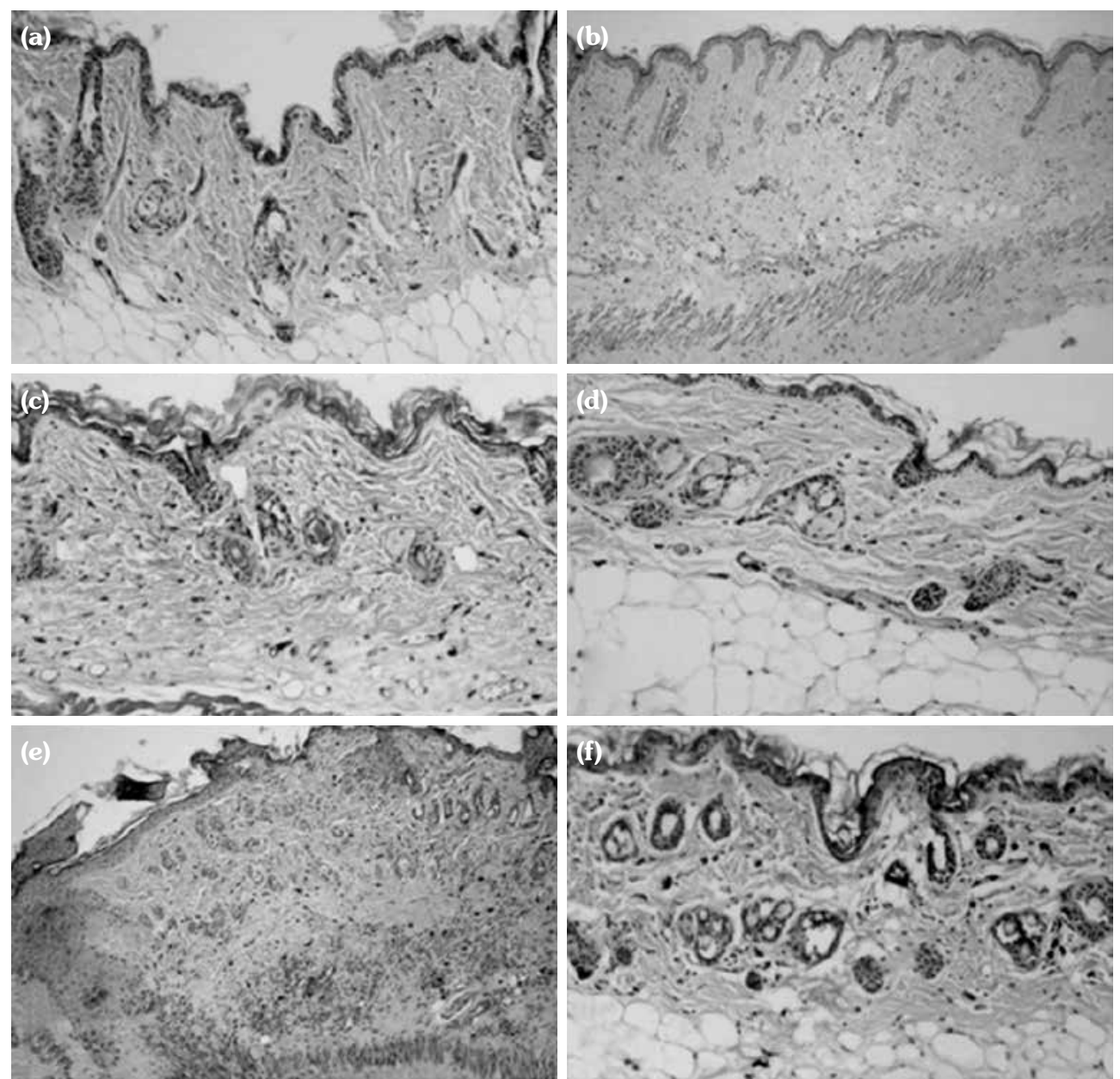

Figure 2. Histopathological appearances of skin sections $(H-E \times 200)$. Histopathological appearances were normal in control groups (a and d). Bleomycin caused thickened collagen bundles in dermis and definite skin fibrosis in sham groups (b and e). Prophylactic and therapeutic applications of octreotide ameliorated dermal fibrosis and decreased infiltrations of inflammatory cells (c and f, respectively). 
Master Mix (Applied Biosystems, Foster City, CA, USA). The results were standardized to the levels of glyceraldehyde 3-phosphate dehydrogenase. The samples were quantified for fibronectin, TGF$\beta 1$, Wnt-2 and axin-1 messenger RNA (mRNA) (Applied Biosystems, Foster City, CA, USA) using the comparative delta $\mathrm{Ct}$, as described in the User's Manual (Applied Biosystems, Foster City, CA, USA).

\section{Statistical analysis}

Statistical evaluations were performed using the IBM SPSS Statistics V22.0 (IBM Corp., Armonk, NY, USA) package program. The data were expressed as mean \pm standard deviation. Kruskal-Wallis one-way analysis of variance and post-hoc Mann-Whitney U test were applied for statistical comparisons. A $p$ value of $<0.05$, after Bonferroni correction for multiple comparisons, was accepted to be significant.

\section{RESULTS}

Dermal fibrosis was observed in the BLM injected mice at the end of third week, while dermal fibrosis was exacerbated at the end of sixth week (Figures 2 and 3). Among the prophylactic application groups, the mean dermal inflammatory cell counts $(34.1 \pm 9.4$ vs. $5.3 \pm 2.1, p=0.003)$ (Figure 3a) and the mean dermal thickness (354.1 \pm 39.8 vs. $189.3 \pm 29.3 \mu \mathrm{m}, \mathrm{p}=0.003$ ) (Figure $3 b$ ) were higher in the sham I group when

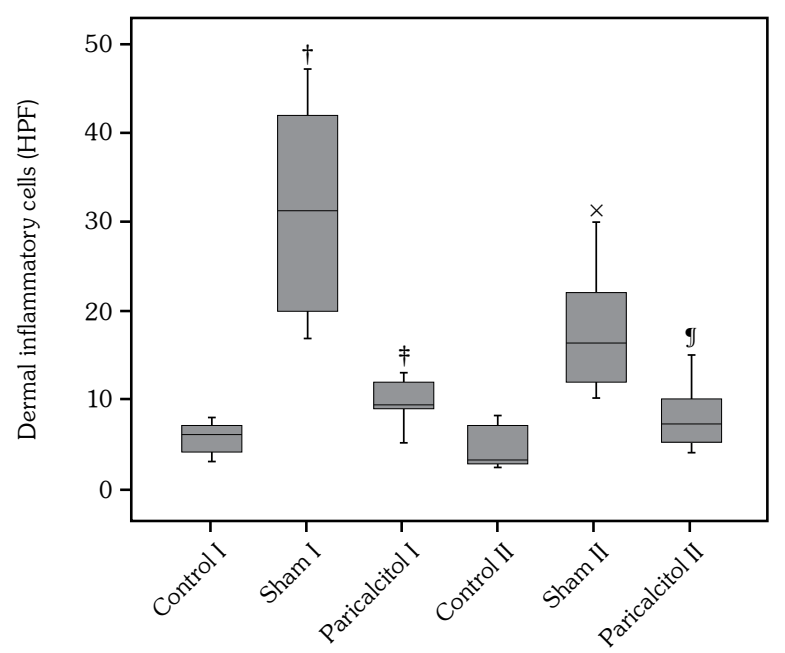

compared to the control I group. At the sixth week, the mean inflammatory cell count $(18.2 \pm 7.5$ vs. $4.4 \pm 1.8, \mathrm{p}=0.001)$ and dermal thickness $(527.1 \pm 98.5$ vs. $149.9 \pm 28.2 \mu \mathrm{m}, \mathrm{p}<0.001)$ were higher in the sham II group compared to the control II group (Figure 3).

Moreover, when compared to the own control group, the BLM injection increased mRNA expressions of fibronectin, TGF- $\beta 1$, Wnt- 2 and axin-1 3.9, 42, 0.8 and 120 folds, respectively at the third week; and 259, 956, 27 and 2,929 folds, respectively at the sixth week (Figure 4).

In the prophylactic paricalcitol group, the mean inflammatory cell count $(34.1 \pm 9.4$ vs. $9.9 \pm 2.6, p=0.001)$ and the mean dermal thickness (354.1 \pm 39.8 vs. $248.9 \pm 50.5 \mu \mathrm{m}, \quad \mathrm{p}=0.007$ ) were decreased when compared to the sham I group (Figures 2 and 3). Moreover, therapeutic paricalcitol applications decreased the mean inflammatory cell count $(18.2 \pm 7.5$ vs. $8.3 \pm 4.1$, $\mathrm{p}=0.005)$ and dermal thickness $(527.1 \pm 98.5$ vs. $319.1 \pm 56.5 \mu \mathrm{m}, \mathrm{p}=0.003)$ when compared to the sham II group (Figure 2).

On the other hand, prophylactic paricalcitol application decreased TGF- $\beta 1$ and axin-1 mRNA expressions ( $p<0.05$ for both) but not fibronectin and Wnt-2 mRNA expressions. However, therapeutic applications decreased fibronectin, TGF- $\beta 1$, Wnt-2 and axin-1 mRNA expressions $(p<0.05$ for all) (Figure 4).

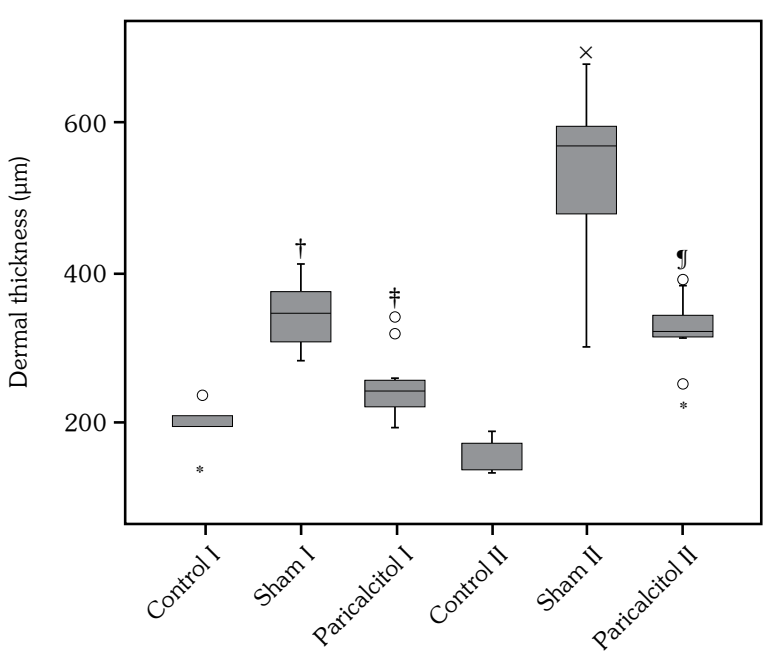

Figure 3. (a) Dermal inflammatory cell counts and (b) dermal thicknesses in the study groups. HPF: High power field; $\dagger \mathrm{p}$ value was $<0.05$, when compared to the Control I group. $\neq p$ value was $<0.05$, when compared to the Sham I group. $\times p$ value was $<0.05$, when compared to the Control II group. \I $p$ value was $<0.05$, when compared to the Sham II group. 
(a)

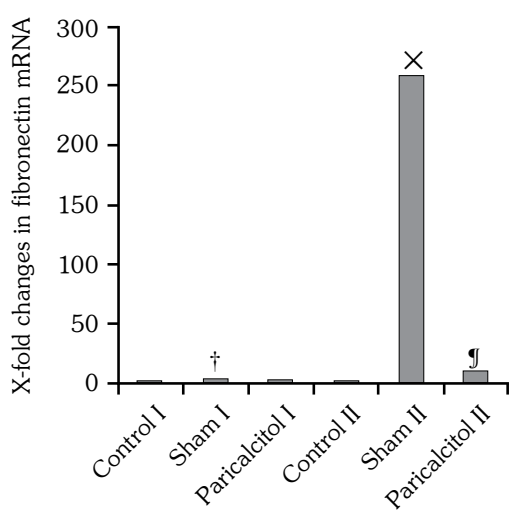

(c)

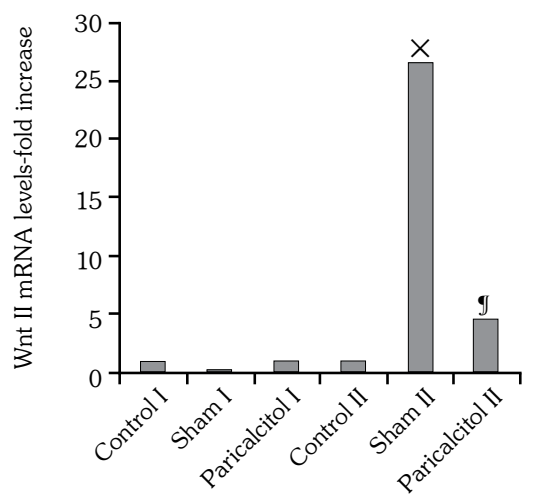

(b)

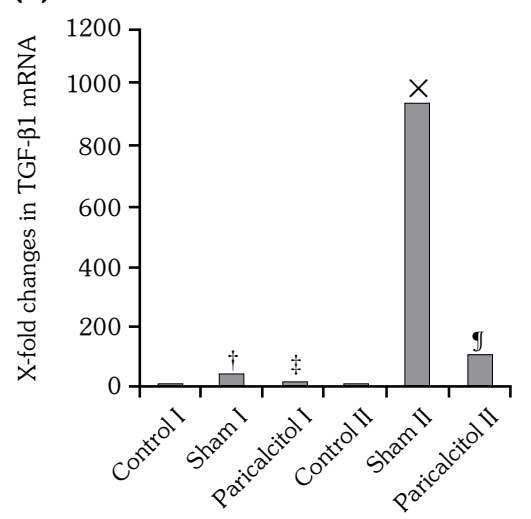

(d)

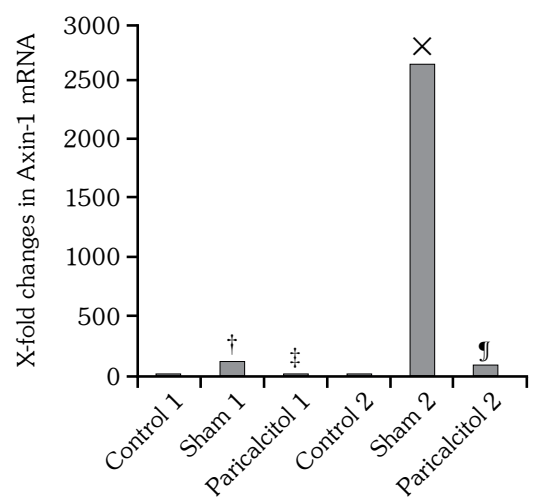

Figure 4. (a) Dermal tissue messenger ribonucleic acid expressions of fibronectin, (b) transforming growth factor-beta 1, (c) Wnt II, and (d) Axin-1. Gene values were normalized to glyceraldehyde 3-phosphate dehydrogenase level; mRNA: Messenger ribonucleic acid; TGF: Transforming growth factor; $\uparrow p$ value was $<0.05$, when compared to the Control I group. $\neq p$ value was $<0.05$, when compared to the Sham I group. $\times p$ value was $<0.05$, when compared to the Control II group. II $\mathrm{p}$ value was $<0.05$, when compared to the Sham II group.

\section{DISCUSSION}

In the present study, the findings of early and late skin fibrosis in an experimental model induced by BLM, and the prophylactic and therapeutic potentials of paricalcitol on skin fibrosis were investigated. Repeated BLM applications led to dermal fibrosis starting at the end of third week and dermal fibrosis was marked at the end of sixth week. On the other hand, this study showed that paricalcitol prevented dermal fibrosis and ameliorated established fibrosis.

Scleroderma is characterized by skin fibrosis. However, its pathogenesis needs to be clarified. BLM induced dermal fibrosis model is one of the experimental scleroderma models. In this study, repeated BLM injections led to dermal fibrosis and increased Wnt- 2 and axin-1 mRNA expressions. On the other hand, paricalcitol treatment ameliorated dermal fibrosis as well as decreasing Wnt-2 and axin-1 mRNA expressions. These results suggest that Wnt/ $\beta$-catenin pathway acts roles in the pathogenesis of dermal fibrosis.

Wnt/ $\beta$-catenin signaling pathway affects embryogenesis and organogenesis via contributing cell-cell interaction and cell transformation. It has been reported that dermal Wnt-2, Wnt$10 \mathrm{~b}$ and Wnt-11 expressions are increased in the experimental scleroderma models. ${ }^{10,20}$ Moreover, in patients with scleroderma, skin Wnt-1 and Wnt-11 expressions have been documented to be increased, while skin DKK1 expression, an inhibitor of Wnt/ $\beta$-catenin signaling pathway, is decreased. ${ }^{11}$ Similarly, increased skin and lung tissue $\beta$-catenin levels ${ }^{11}$ suggest that $\mathrm{Wnt} / \beta$-catenin signaling pathway is activated in scleroderma. 
Wnt-3 and Wnt-10 $\beta$ applications have been demonstrated to induce dermal fibrosis in in vivo studies. Moreover, in in vitro setting, Wnt-3 application has induced the expression of smooth muscle actin, an indicator of myofibroblastic activity. At the end of these experiments, the researchers suggest that activated Wnt/ $\beta$-catenin pathway leads to fibrosis through its effects on the fibroblastic activity and the transformations of the non-fibroblastic cells to fibroblastic cells. ${ }^{10,13}$ In this study, BLM applications induced dermal fibrosis and increased dermal Wnt- 2 and axin-1 mRNA expressions. Increased Wnt- 2 and axin- 1 mRNA expressions suggest that $\mathrm{Wnt} / \beta$-catenin pathway is activated in dermal fibrosis.

Paricalcitol, 19-nor-1,25-hydroxivitamin D2, is a synthetic analog of vitamin D. ${ }^{21}$ Paricalcitol has ameliorated renal injury via inhibiting proinflammatory cytokine release, monocyte/ macrophage infiltration and myofibroblastic activity, and decreasing the expression of fibronectin, TGF- $\beta 1$, type-I and -III collagen. ${ }^{18}$ In this study, paricalcitol decreased inflammatory cell count and dermal thickness in both early and late phases of BLM induced dermal fibrosis.

The accumulations of collagen and other ECM molecules lead to fibrosis, although the pathogenesis is not fully elucidated, in scleroderma. $^{2}$ One cause of excessive ECM production is activated fibroblasts. ${ }^{3,4}$ TGF- $\beta$ acts a prominent role on the activity of myofibroblast and the production of ECM molecules. ${ }^{5}$ In this study, the increased dermal TGF- $\beta 1$ mRNA expression was detected in the dermal fibrosis model induced by BLM.

Transforming growth factor-beta is a pathogenic actor in renal fibrosis, too. In the cisplatin induced renal injury model, paricalcitol is reported to decrease apoptosis, renal injury and renal fibrosis. ${ }^{22}$ Moreover, paricalcitol is documented to abolish the infiltration of $\mathrm{T}$ lymphocytes and macrophages in obstructive nephropathy model. ${ }^{18}$ It has been shown that paricalcitol leads to anti-inflammatory and antifibrotic effects and ameliorates renal injury under in vitro conditions, in the cyclosporine induced renal injury model. ${ }^{23}$ Paricalcitol has been demonstrated to deplete fibrosis induced by TGF- $\beta 1$ under in vitro conditions in human renal epithelial cells. ${ }^{24}$
In this study, paricalcitol prevented and ameliorated dermal fibrosis. The anti-fibrotic action of paricalcitol can be related with its effects on TGF- $\beta$. Paricalcitol applications decreased dermal TGF- $\beta 1$ mRNA expression, in addition to dermal fibrosis. On the other hand, it is known that vitamin $\mathrm{D}$ competitively inhibits the interaction of TCF-4 and $\beta$-catenin through VDR. ${ }^{15,16}$ Thus, paricalcitol, a nonhypercalcemic synthetic vitamin $\mathrm{D}$ analog, depletes the activity of Wnt/ $\beta$-catenin pathway. ${ }^{17}$ Therefore, it can be concluded that anti-fibrotic effect of paricalcitol on dermal fibrosis is related with its effects on Wnt/ $\beta$-catenin pathway. Surely, in this study, paricalcitol decreased dermal Wnt-2 and axin-1 mRNA expressions in addition to dermal thickness.

The present study has several limitations. Firstly, the hypothesis of the study could also be investigated under an in vitro setting. Secondly, the hypothesis could be analyzed in another experimental model. Lastly, TGF- $\beta 2$ and 3 are important in the pathogenesis of scleroderma in addition to TGF- $\beta 1$. Thus, TGF- $\beta 2$ and 3 could be analyzed.

In conclusion, in the BLM induced dermal fibrosis model, increased axin-1 and Wnt- 2 mRNA expressions suggest that Wnt/ $\beta$-catenin signaling pathway is active in dermal fibrosis. Moreover, paricalcitol has an anti-fibrotic potential and this effect may be associated on Wnt/ $\beta$-catenin signaling pathway. These results suggest that paricalcitol may be a therapeutic agent for scleroderma. Moreover, Wnt/ $\beta$-catenin signaling pathway may be a therapeutic in scleroderma.

\section{Declaration of conflicting interests}

The authors declared no conflicts of interest with respect to the authorship and/or publication of this article.

\section{Funding}

This study was supported by Firat University Scientific Research Projects Coordination Unit (FUBAP).

\section{REFERENCES}

1. Varga J, Abraham D. Systemic sclerosis: a prototypic multisystem fibrotic disorder. J Clin Invest 2007;117:557-67.

2. Denton CP, Black CM, Abraham DJ. Mechanisms and consequences of fibrosis in systemic sclerosis. Nat Clin Pract Rheumatol 2006;2:134-44. 
3. Krieg T, Abraham D, Lafyatis R. Fibrosis in connective tissue disease: the role of the myofibroblast and fibroblast-epithelial cell interactions. Arthritis Res Ther 2007;9:4.

4. Postlethwaite AE, Shigemitsu H, Kanangat S. Cellular origins of fibroblasts: possible implications for organ fibrosis in systemic sclerosis. Curr Opin Rheumatol 2004; 16:733-8.

5. Varga J, Pasche B. Transforming growth factor beta as a therapeutic target in systemic sclerosis. Nat Rev Rheumatol 2009;5:200-6.

6. Logan CY, Nusse R. The Wnt signaling pathway in development and disease. Annu Rev Cell Dev Biol 2004;20:781-810.

7. van Amerongen R, Nusse R. Towards an integrated view of Wnt signaling in development. Development 2009;136:3205-14.

8. Cadigan KM, Liu YI. Wnt signaling: complexity at the surface. J Cell Sci 2006;119:395-402.

9. Bayle J, Fitch J, Jacobsen K, Kumar R, Lafyatis R, Lemaire R. Increased expression of Wnt2 and SFRP4 in Tsk mouse skin: role of Wnt signaling in altered dermal fibrillin deposition and systemic sclerosis. $\mathrm{J}$ Invest Dermatol 2008;128:871-81.

10. Wei J, Melichian D, Komura K, Hinchcliff M, Lam AP, Lafyatis $\mathrm{R}$, et al. Canonical Wnt signaling induces skin fibrosis and subcutaneous lipoatrophy: a novel mouse model for scleroderma? Arthritis Rheum 2011;63:1707-17.

11. Akhmetshina A, Palumbo K, Dees C, Bergmann C, Venalis $\mathrm{P}$, Zerr $\mathrm{P}$, et al. Activation of canonical Wnt signalling is required for TGF- $\beta$-mediated fibrosis. Nat Commun 2012;3:735.

12. Farina G, Lafyatis D, Lemaire R, Lafyatis R. A fourgene biomarker predicts skin disease in patients with diffuse cutaneous systemic sclerosis. Arthritis Rheum 2010;62:580-8.

13. Wei J, Fang F, Lam AP, Sargent JL, Hamburg E, Hinchcliff $\mathrm{ME}$, et al. Wnt/ $\beta$-catenin signaling is hyperactivated in systemic sclerosis and induces Smad-dependent fibrotic responses in mesenchymal cells. Arthritis Rheum 2012;64:2734-45.

14. Lemaire R, Farina G, Bayle J, Dimarzio M, Pendergrass SA, Milano A, et al. Antagonistic effect of the matricellular signaling protein CCN3 on TGF-beta- and Wnt-mediated fibrillinogenesis in systemic sclerosis and Marfan syndrome. J Invest Dermatol 2010;130:1514-23.

15. Pálmer HG, González-Sancho JM, Espada J, Berciano MT, Puig I, Baulida J, et al. Vitamin D(3) promotes the differentiation of colon carcinoma cells by the induction of E-cadherin and the inhibition of betacatenin signaling. J Cell Biol 2001;154:369-87.

16. Shah S, Islam MN, Dakshanamurthy S, Rizvi I, Rao $\mathrm{M}$, Herrell R, et al. The molecular basis of vitamin D receptor and beta-catenin crossregulation. Mol Cell 2006;21:799-809.

17. He W, Kang YS, Dai C, Liu Y. Blockade of Wnt/ $\beta$-catenin signaling by paricalcitol ameliorates proteinuria and kidney injury. J Am Soc Nephrol 2011;22:90-103.

18. Tan X, Li Y, Liu Y. Paricalcitol attenuates renal interstitial fibrosis in obstructive nephropathy. J Am Soc Nephrol 2006;17:3382-93.

19. Yamamoto T. Animal model of sclerotic skin induced by bleomycin: a clue to the pathogenesis of and therapy for scleroderma? Clin Immunol 2002;102:209-16.

20. Bayle J, Fitch J, Jacobsen K, Kumar R, Lafyatis R, Lemaire R. Increased expression of Wnt2 and SFRP4 in Tsk mouse skin: role of Wnt signaling in altered dermal fibrillin deposition and systemic sclerosis. J Invest Dermatol 2008;128:871-81.

21. Goldenberg MM. Paricalcitol, a new agent for the management of secondary hyperparathyroidism in patients undergoing chronic renal dialysis. Clin Ther 1999;21:432-41.

22. Park JW, Cho JW, Joo SY, Kim CS, Choi JS, Bae $\mathrm{EH}$, et al. Paricalcitol prevents cisplatin-induced renal injury by suppressing apoptosis and proliferation. Eur J Pharmacol 2012;683:301-9.

23. Piao SG, Song JC, Lim SW, Chung BH, Choi BS, Yang CW. Protective effect of paricalcitol on cyclosporine-induced renal injury in rats. Transplant Proc 2012;44:642-5.

24. Nolan KA, Brennan EP, Scholz CC, Cullen C, Ryan A, Taylor CT, et al. Paricalcitol protects against TGF- $\beta 1$-induced fibrotic responses in hypoxia and stabilises HIF- $\alpha$ in renal epithelia. Exp Cell Res 2015;330:371-81. 